\title{
Feature Extraction in Local Spectrum Sensing for Next Generation Cognitive Radios - A Review
}

\author{
Antoni Ivanov \\ Department of Communication Networks, Technical University of Sofia, \\ Sofia, 1000, Bulgaria \\ E-mail: astivanov@tu-sofia.bg
}

Received 01 October 2019; Accepted 22 January 2020;

Publication 31 January 2020

\begin{abstract}
In the context of the currently developed networks for the realization of future communications, the concept of Cognitive Radio (CR) has a significant place. Its implementation in modern ultra-dense networks (UDN) requires the development of novel and improved adaptive solutions for the main functionalities of a CR system. This technology has a great potential for solving the significant spectrum underutilization issue which has been established as characteristic for the traditional communication networks and hence, the continual and substantive research efforts in the recent years. The principal challenge for CRs is the optimization of spectrum utilization without creating unwanted interference for the incumbent (primary) users. Thus, a significant portion of the research is directed towards the vital spectrum sensing functionality. This paper reviews the potential uses of CR in UDN as well as the main approaches for modeling of spectrum sensing with respect to signal detection. The review focuses on local spectrum sensing techniques and presents the strengths and weaknesses of their mathematical definition. Specific attention is given to the channel models which are considered in the literature and to what kinds of features are extracted from the received signal to achieve accurate detection.
\end{abstract}

Journal of Mobile Multimedia, Vol. 15_1\&2, 111-140.

doi: $10.13052 / \mathrm{jmm} 1550-4646.15126$

(c) 2020 River Publishers 
Keywords: Cognitive radio, ultra-dense networks, spectrum sensing, signal detection, feature extraction.

\section{Introduction}

The application of wireless communications in many of man's everyday activities has been established through the astonishing progress of computation technologies in the recent decades. Their further consolidation is only a matter of time due to the various intelligent devices and applications for the current and future uses of the consumer. The wireless technologies themselves and the freedom and flexibility of use that they offer determine the dynamic formation of new applications, such as virtual reality, holoportation, much more reliable mobile communications in the presence of high volume of traffic and crowds, Internet of Things (IoT), Internet of Vehicles (IoV), Device to Device (D2D) communication, eHealth, Machine to Machine (M2M) communication [1-3]. All of these technologies are changing the way humans interact with each other and their environment, providing ongoing connectivity and communication with any system, device or person from any location, anytime, using apparent infinite capacity, realized through the efficient use of the telecommunications infrastructure [4]. Recent forecasts estimate the growth of total mobile traffic as a systematic increase of $40 \%$ per year over the next three years, with the most significant part of it focused on the consumption generated by the Asian market [5]. At the same time, a significant increase in IoT and M2M communications is expected. In order to provide connectivity to such a big number of devices, the $5 \mathrm{G}$ standards are required to achieve speeds of the order of several Gbps, latency of around $1 \mathrm{~ms}$, tens of thousands of user devices per $\mathrm{km}^{2}$, improved Quality of Service (QoS) and implementation flexibility [3]. These specifications are envisioned to be fulfilled by the UDNs. However, there is a need of a new paradigm in the organization and design of wireless communications, due to approaching of the Shannon theoretical limit and the saturation of the frequency channels in the $300 \mathrm{MHz}-6 \mathrm{GHz}$ band [3]. On the other hand, together with the limitation and high cost of these frequency resources, largescale practical studies of the real spectrum utilization in this range, show that even the most occupied channels (those used for mobile communications) have an average duty cycle below 50\% [6-8]. In his seminal work examining wireless communications with added intelligence [9], S. Haykin summarizes that even in an urban environment, there are frequency channels that are either partially utilized or completely free, most of the time. There are a few studies 
(namely [10-15]) on the application of CR principles in UDNs. The cognitive functionality of $\mathrm{CR}$ devices allows efficient use of the unutilized spectrum and collaboration between multiple networks operating in the same or adjacent wide bandwidths, thus providing high throughput communications secure from interference between the primary users (PU) and the cognitive, i.e. secondary users (SU). Thus, implementing functionalities from the CR in UDNs, allows the system to operate autonomously from traditional communication networks (e.g. television broadcasting), which are based on macro and micro cells. Some recent UDN models with their potential for extension through cognitive functionalities are outlined in Table 1 . The prospect of integrating cognitive concepts into the developing dense 5G networks can lead to many novel solutions for accommodating them to operate in the

Table 1 A brief review of notable works in the field of UDN with respect to the possibilities of applying CR concepts

\begin{tabular}{|c|c|c|c|}
\hline & $\begin{array}{c}\text { Application/ } \\
\text { Purpose of the Study }\end{array}$ & Experimental Setup & Possible Extensions \\
\hline [15] & $\begin{array}{l}\text { Algorithm for choosing } \\
\text { a BS and avoidance of } \\
\text { inter-cell interference } \\
\text { and throughput increase. }\end{array}$ & $\begin{array}{l}\text { Heterogeneous network } \\
\text { comprised of macro- and } \\
\text { small cells, the resource } \\
\text { allocation and BS choice } \\
\text { is performed in the cloud. }\end{array}$ & $\begin{array}{l}\text { Applying the proposed } \\
\text { strategy in autonomous } \\
\text { CR networks. }\end{array}$ \\
\hline [16] & $\begin{array}{l}\text { User protection from } \\
\text { physical layer attack in } \\
\text { D2D. }\end{array}$ & $\begin{array}{l}\text { D2D network with base } \\
\text { stations (BSs), user } \\
\text { devices and eaesdroppers } \\
\text { with Poisson distribution. }\end{array}$ & $\begin{array}{l}\text { Incorporation of CR } \\
\text { networks into the } \\
\text { scenario. }\end{array}$ \\
\hline [17] & $\begin{array}{l}\text { Resource distribution in } \\
\text { a D2D network. }\end{array}$ & $\begin{array}{l}\text { D2D network with BSs, } \\
\text { mobile users and D2D } \\
\text { nodes with Poisson } \\
\text { distribution. Every } \\
\text { channel can be allocated } \\
\text { to a specific user alone. }\end{array}$ & $\begin{array}{l}\text { Incorporation of CR } \\
\text { networks into the } \\
\text { scenario. }\end{array}$ \\
\hline$[18]$ & $\begin{array}{l}\text { Adaptive organization of } \\
\text { femto-BSs inside the } \\
\text { region of service of a } \\
\text { macro-BS, to avoid } \\
\text { interference. }\end{array}$ & $\begin{array}{l}\text { The femto-BSs are } \\
\text { Poisson-distributed and } \\
\text { share common frequency } \\
\text { channels with the } \\
\text { macro-BS. They all } \\
\text { belong to the same } \\
\text { network. }\end{array}$ & $\begin{array}{l}\text { The scenario can be } \\
\text { adapted to incorporate the } \\
\text { CR concept by } \\
\text { introducing secondary } \\
\text { femto-cells which are not } \\
\text { incumbent to the } \\
\text { macro-BS's spectrum. }\end{array}$ \\
\hline
\end{tabular}


Table 1 Continued

\begin{tabular}{|c|c|c|c|}
\hline & $\begin{array}{c}\text { Application/ } \\
\text { Purpose of the Study }\end{array}$ & Experimental Setup & Possible Extensions \\
\hline [19] & $\begin{array}{l}\text { Cooperative resource } \\
\text { allocation between a } \\
\text { macro-cell and small cells } \\
\text { of a UDN through } \\
\text { cognitive capabilities. The } \\
\text { allocation is done in time } \\
\text { and in spatial domains. }\end{array}$ & $\begin{array}{l}\text { Determination of the } \\
\text { maximal number of } \\
\text { cognitive small cells } \\
\text { which can be deployed in } \\
\text { the coverage region of the } \\
\text { macro-cell without } \\
\text { intolerable interference } \\
\text { levels. }\end{array}$ & $\begin{array}{l}\text { Incorporation of dynamic } \\
\text { access of the CR users to } \\
\text { the spectrum, to provide } \\
\text { autonomy between the } \\
\text { UDN and the macro-cell's } \\
\text { network. }\end{array}$ \\
\hline [20] & $\begin{array}{l}\text { Resource allocation in } \\
\text { UDN with added } \\
\text { cognition through } \\
\text { hyper-graph theory. } \\
\text { Network optimization to } \\
\text { achieve user association. }\end{array}$ & $\begin{array}{l}\text { Distributed network for } \\
\text { which the Access Points } \\
\text { (APs) of the UDN operate } \\
\text { within the range of one } \\
\text { macro-cell and share } \\
\text { resources in cooperation } \\
\text { with it. }\end{array}$ & $\begin{array}{l}\text { Extending the problem to } \\
\text { autonomous spectrum } \\
\text { sharing between the UDN } \\
\text { and the network of the } \\
\text { macro-cell. }\end{array}$ \\
\hline [21] & $\begin{array}{l}\text { Resource distribution } \\
\text { among a macro-cell and } \\
\text { APs operating in the } \\
\text { millimeter wave-range. }\end{array}$ & $\begin{array}{l}\text { Distributed network for } \\
\text { which the APs of the } \\
\text { UDN operate within the } \\
\text { range of one macro-cell } \\
\text { and share resources in } \\
\text { cooperation with it. }\end{array}$ & $\begin{array}{l}\text { Extending the problem to } \\
\text { autonomous spectrum } \\
\text { sharing between the UDN } \\
\text { and the network of the } \\
\text { macro-cell in the } \\
\text { millimeter wave-range. }\end{array}$ \\
\hline [22] & $\begin{array}{l}\text { Resource distribution } \\
\text { between different mobile } \\
\text { operators in unlicensed } \\
\text { spectrum. }\end{array}$ & $\begin{array}{l}\text { Spectrum sharing among } \\
\text { the network nodes through } \\
\text { game theory and } \\
\text { availability of full channel } \\
\text { state information (CSI). }\end{array}$ & $\begin{array}{l}\text { Incorporation of dynamic } \\
\text { spectrum access and } \\
\text { autonomous spectrum } \\
\text { sharing between the } \\
\text { different networks. }\end{array}$ \\
\hline [23] & $\begin{array}{l}\text { Topology design for } \\
\text { UDNs. }\end{array}$ & $\begin{array}{l}\text { Determination of the APs' } \\
\text { range and the optimal } \\
\text { number of users with } \\
\text { consideration of the } \\
\text { propagation conditions. }\end{array}$ & $\begin{array}{l}\text { Topology design for dense } \\
\text { CR networks. }\end{array}$ \\
\hline
\end{tabular}

congested traditional bands. This will also make the transition to 5G and beyond, smoother because the current telecommunications standards will be able to seamlessly coexist with the new ones. It is through the spectrum sensing functionality that this can be achieved. Thus, it is the purpose of this article to present the main types of sensing methods with their characteristics and design challenges. They can be used as a blueprint for the development of novel CR-based UDN architectures. This work reviews a variety of notable 
algorithms for local spectrum sensing (single device is considered) from the point of view that in order for the CR network to utilize the spectrum efficiently, it requires rigorous sensing to be performed in every individual SU. The rest of the paper is organized in the following manner. Section II briefly describes the general way of operation of a CR device with respect to the dynamic access of the PU's spectrum. Then, the general structure of a signal detector and the fundamental concepts of its operation are briefly described in Section III. The review of the spectrum sensing methods is given in Section IV. Finally, Section V contains a summary and future research challenges based on the observed tendencies.

\section{Cognitive Radio Networks in the Context of 5G}

The introduction of the software-defined radio (SDR) facilitates the provision of additional services when unutilized PU channels are available. The well-established definition of the $\mathrm{CR}$ is as follows [9]: "Cognitive radio is an intelligent wireless communication system that is aware of its surrounding environment (i.e., outside world), and uses the methodology of understanding-by-building to learn from the environment and adapt its internal states to statistical variations in the incoming radio frequency (RF) stimuli by making corresponding changes in certain operating parameters (e.g., transmit power, carrier frequency, and modulation strategy) in real-time". According to this formulation, a CR device learns from the characteristics of the RF environment (noise level, traffic statistics, unused portions of the spectrum), predicts the status of these characteristics and adjusts its transmission parameters to achieve efficient use of spectrum (correct detection of spectrum holes and their utilization) and reliable communications for both PUs and SUs. In other words, the CR devices are required to detect spectrum holes (unutilized frequency bands) with reliability of $90 \%$ at signal-to-noise ratio of $-20 \mathrm{~dB}$ [24], and utilize the spectrum for as long as it is vacant from the point of view of the PU.

The SU must immediately release the spectrum if the PU initiates transmission in it, to avoid interfering with the primary communications. SU should also be highly effective in carrying out the assessment process, because a wrong conclusion on the channel occupancy can either lead to interference for the incumbent users of the spectrum or a missed opportunity to utilize the available resource. For these reasons, the ability of the CR to evaluate (or monitor the spectrum) is a major component of the device in the context of 5G and UDN. It is also essential for the implementation of the four 


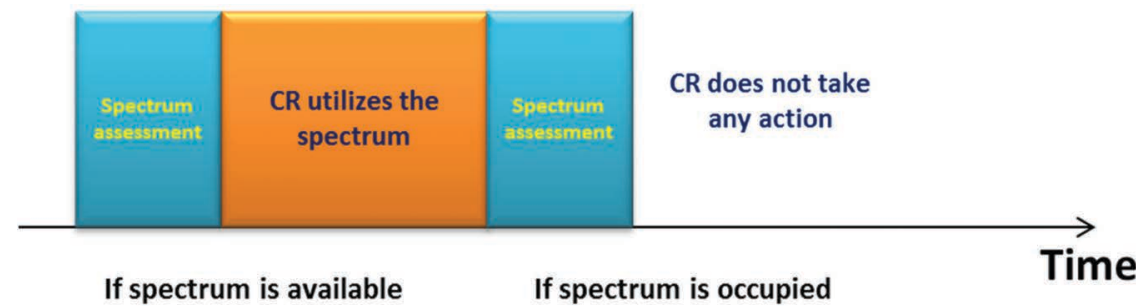

Figure 1 Operating model of a half-duplex CR device.

structural operational features of the $5 \mathrm{G}$ systems - cognition, cooperation, network virtualization and redistribution of network traffic [25].

An overview of the operation of an individual CR device is presented in Figure 1. It can be divided into frames (slots, cycles), during which the SU performs its cognitive functions (monitoring the spectrum and utilizing the available bands). Each frame begins with spectrum evaluation (sensing, assessment) to allow the device to determine which frequency channels are free of PU transmissions, if any. In case that an unoccupied spectrum chunk is identified, the SU may use it for its transmission within a specified interval, which may be accepted as the remainder of the frame. If all frequency channels are occupied by the PU, then the CR will have no access to the spectrum and goes into inactive state. Alternatively, it may continue to monitor the frequency resource because it may be vacated before the end of the current frame.

It should be noted that this review deals with only half-duplex transceiver cases, i.e. those which operate with only one transceiver module and must alternate between transmission and reception (respectively, spectral monitoring), according to the classical operating model of the CR devices described in Figure 1. In recent years, a certain amount of interest among the scientific community has been given to full-duplex transceivers for CR applications. In these cases, the availability of two transceivers in one device allows simultaneous monitoring and utilization of the spectrum [26]. This provides the CR drive with much more flexibility due to the avoidance of problems related to spectrum underutilization and the risk of interference to the PU. The addition of a second transceiver module, working in parallel with the first, however, significantly changes the cognitive user equipment's features and increases its cost. For this reason, full-duplex transceivers and the specifics of their implementation are beyond the scope of this paper. 


\section{Signal Detectors in Cognitive Radio Networks}

The spectrum sensing function generally aims to differentiate the PU signal from the noise with sufficient accuracy, i.e. it is a detection task. A summary diagram of the signal detector is shown in Figure 2. A CR device performs a measurement in a bandwidth of $W \mathrm{~Hz}$ over a sensing period of $t_{S}$ seconds in which $N=2 t_{S} W$ samples are obtained at the output of the analog-todigital converter (ADC). The received signal y is subjected to processing tied to the specific sensing method in order to extract the necessary features (for example, signal level, noise level, eigenvalues, etc.) that will allow the detector to correctly evaluate with certain probability, the presence of the PU signal in the band. Finally, the detector makes a decision.

Traditionally, the signal detector's task is described through the differentiation between two hypotheses - null $H_{0}$ (the band contains only noise, i.e. it is free from PU transmissions) and alternative $H_{1}$ (the PU signal is present). Equation (1) expresses these relations:

$$
\begin{aligned}
& H_{0}: y=n \\
& H_{1}: y=x+n .
\end{aligned}
$$

The sensing function must choose one of the two hypotheses based on the test statistic $\Lambda$ resulting from the processing of $\mathrm{y}$, which is compared to the value of the decision threshold $\lambda$ previously calculated. It characterizes the noise level and, accordingly, if $\Lambda$ has a higher value than the threshold, the alternative hypothesis $H_{1}$ is validated:

$$
\Lambda(y) \gtreqless \lambda
$$

The detector's efficiency is determined by the adequate choice of the threshold. It has to be chosen such that the detector will be able to discriminate between the two hypotheses with sufficient accuracy [27]. The difficulty in making this decision increases substantially as the noise power becomes comparable or higher than that of the signal. Such case is illustrated in Figure 3. The probability distribution of $H_{0}$ is illustrated by the area marked by the blue line, whereas that of the alternative - by the red one. The signal

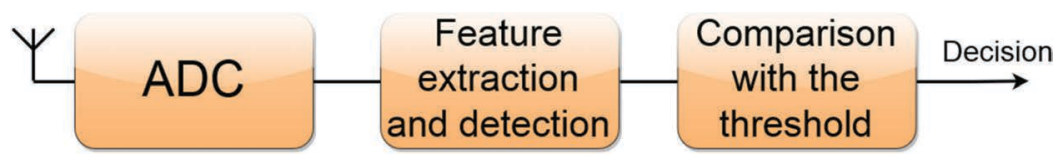

Figure 2 Generalized scheme of the spectrum sensing operations in CR. 


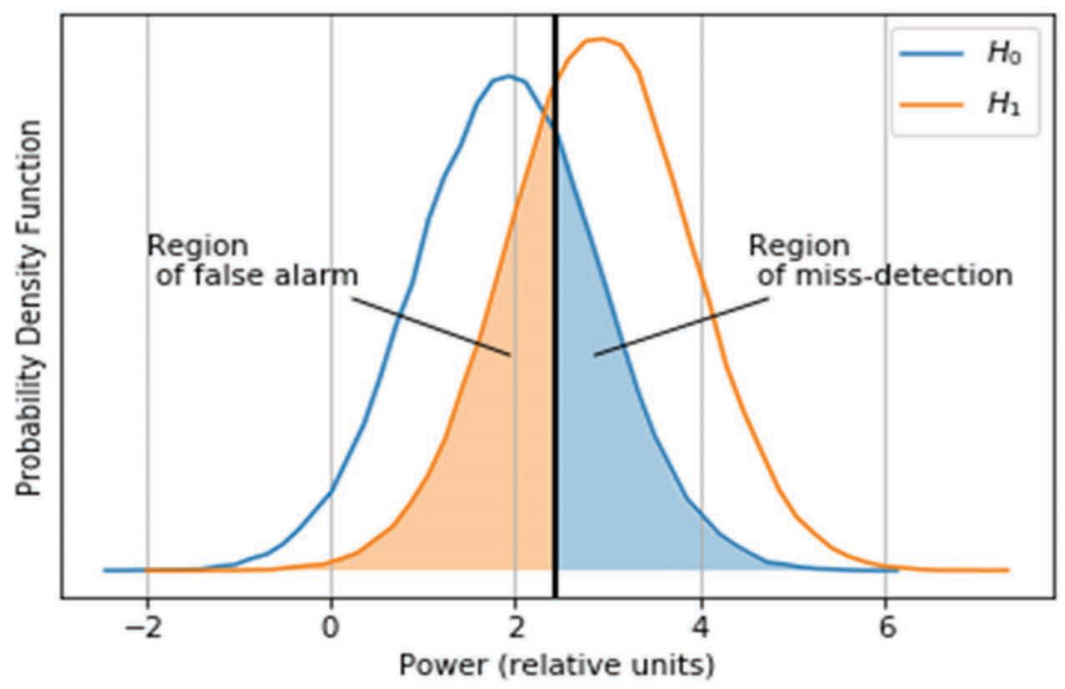

Figure 3 Generalized scheme of the spectrum sensing operations in CR.

power is only 1.5 times higher than that of the noise. Thus, the decision threshold's setting becomes a trade-off between a certain probability of missdetection $P_{M D}=p\left(H_{0} \mid H_{1}\right)$ (the area colored in blue) and a probability of false alarm $P_{F A}=p\left(H_{1} \mid H_{0}\right)$ (in orange). This figure illustrates the distribution ratio of the two probabilities of error when the threshold (the bold black vertical line) is set such that they are both around 50\%. The detector's purpose is to find the optimal balance for the choice of threshold with one of these probabilities set as an initial condition so as to maximize the probability of detection $P_{D}=p\left(H_{1} \mid H_{1}\right)$. Analytically, this process is most often derived by using the Neyman-Pearson criterion even though the Bayesian method is used for that purpose if the assumed channel models are more complex [27].

\section{Detection of Features in Spectrum Sensing}

Spectrum sensing is a core element of a CR device, as it enables the user equipment to operate in shared spectrum conditions with the PU. The time during which a particular spectrum chunk is not used can be very short, and therefore the SU must be able to quickly determine whether that chunk is free or not. SU needs to be highly effective in performing this evaluation process, as the wrong conclusion can either lead to interference for users incumbent to the spectrum or lose the ability to utilize the available band. 
The primary purpose of the signal detector to monitor is the precision of decision making according to the theoretical principles set out in Section II. There are several main types of sensing methodologies depending on the character of processing that is required for obtaining the test statistic and/or decision threshold, which are reviewed in this Section.

\subsection{Energy Detectors}

This sensing approach is the easiest to define mathematically and in many cases, the most efficient in terms of computational complexity. For these reasons it is also the most commonly used. In a general sense, this detector finds the overall received samples' power (the sum of their squares) and compares it to a pre-calculated threshold, which represents the maximum noise power in a given bandwidth:

$$
\Lambda_{E D}=\sum_{i=0}^{N-1}\left|y[i]^{2}\right| \gtreqless \lambda .
$$

A general schematic of the operation of this detector is given in Figure 4.

The main disadvantages of this detector are the difficulty in determining the decision threshold, and the inability of the PU signal to be differentiated from other signals, due to the fact that the only feature taken into account in the test statistics is the power of the received signal. The threshold value is determined provided that the noise power in the desired frequency range is known a priori. This drawback can be overcome by introducing an adaptive threshold selection depending on other parameters, such as SNR. Subsequently, from the second major weakness of the ED, the potential for recognition of the PU signal is significantly aggravated at a noise level which is close to or greater than that of the signal. As it was mentioned in Section II, achieving a trade-off between the contradicting $P_{D}$ and $P_{F A}$ involves setting one of them as a parameter, so that the other can be derived from it. Usually this is the probability of false alarm (constant false alarm rate method, CFAR). The general form of the expressions for the threshold and

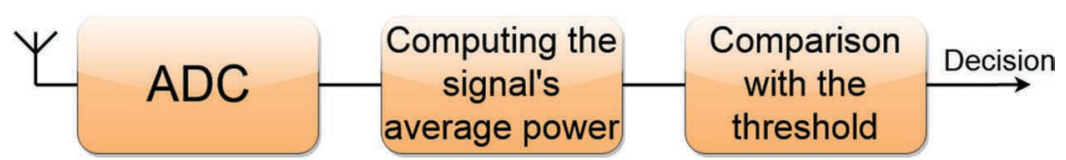

Figure 4 Generalized scheme of the operations in energy detection-based spectrum sensing. 
probability of detection, derived using this approach, is [28]:

$$
\begin{aligned}
\lambda & =\sigma_{n}^{2}\left(\frac{Q^{-1}\left(\bar{P}_{F A}\right)}{\sqrt{N}}+1\right), \\
P_{D} & =Q\left(\frac{1}{\sqrt{2 \gamma+1}}\left(Q^{-1}\left(\bar{P}_{F A}\right)-\sqrt{N} \gamma\right)\right),
\end{aligned}
$$

where $\sigma_{n}^{2}$ is the noise variance ${ }^{1} \gamma=h^{2} \sigma_{s}^{2} / \sigma_{n}^{2}$ is the signal-to-noise ratio, $\sigma_{s}^{2}$ is the signal variance, $h$ is the channel attenuation, $N$ is the number of samples, $\bar{P}_{F A}$ is the set value of the probability of false alarm, and $Q($.$) is$ complimentary distribution function: $Q(x)=\frac{1}{2 \pi} \int_{x}^{\infty} \exp \left(-\frac{t^{2}}{2}\right) \mathrm{d} t$. Several studies have built on top of the traditional ED by applying a threshold which varies with the SNR [29] or differentiation between various received signal levels through multiple thresholds [30,31]. This way the detector's reliability is improved in the areas of intersection between the distributions of the two hypotheses (see Figure 3). The issue of noise uncertainty which indicates the fluctuations in the noise variance that leads to a deterioration of the detector's performance is also considered.

An important concept in the theory of energy detectors is the so called "SNR wall" introduced in [32]. It is defined as such a value of the received signal power that is lower than the noise uncertainty, so that reliable signal detection cannot be achieved even if the sensing time is increased. This phenomenon is addressed in some studies that simulate a low SNR environment $([33,34])$ by utilizing a more rigorous sensing algorithm in case the noise uncertainty is too significant. A more comprehensive solution is proposed in [35]. It is based on the estimated noise power method which readjusts the amount of noise-only (the PU is absent from the spectrum) samples such that the estimator's variance declines. Thus, the SNR wall is alleviated for any particular sensing period (and consequently, number of samples).

A significant portion of the research $([29,36-38])$ related to ED is devoted to the traditional detector model's extension to develop its adaptability. In real-time experiments-based studies such as $[36,39,40]$ it is common that the decision threshold is derived from empirical measurements of the spectrum when the PU transmitter is not active. Proposed modifications are related to the dynamic readjustment of the decision threshold, the comparison of its performance with other sensing methods, the received signal's decomposition into its frequency components, finding the optimal sensing time against the

\footnotetext{
${ }^{1}$ The signal and the noise are both generally assumed to be Gaussian-distributed.
} 
a priori probability of the spectrum being available $p\left(H_{0}\right)$. Studies such as those presented in $[33,41-46]$ introduce an important dimension to the design of ED, namely, they add more realistic propagation conditions to the detector's analytical model. The method proposed in [33] identifies the PU signal under Rayleigh fading and unknown variance of the Gaussian-distributed noise, and in addition to the probability of detection, the spectrum utilization coefficient $^{2}$ is evaluated. A comprehensive analysis of low SNR ED $(<0 \mathrm{~dB})$ for Rayleigh and Nakagami fading is presented in [41]. In addition to designing the detector itself for efficient operation under these conditions, analysis has allowed the definition of optimal threshold such that $P_{D}$ and $P_{F A}$ are guaranteed within certain limits. The authors of [42] consider the case of signal detection with unknown distribution, Gaussian noise and three fading models - Nakagami, Rice and Rayleigh. The decision threshold is determined by the CFAR method. A Gamma fading model (also known as $\alpha-\mu$ fading), along with Gaussian noise, is discussed in [47]. The detector is defined using the Bayesian approach [27, 48], which combines the probabilities of the two wrong decisions $P_{F A}$ and $P_{M D}$ through using the a priori probability $p\left(H_{0}\right)$ of having a spectrum free from PU transmissions and the threshold value. This work is further extended in [43] which considers a generalized fading model termed $\alpha-\kappa-\mu$, which fits experimental measurements well. The study in [44] allows for Nakagami fading, Gaussian noise and shadowing, while the received signal follows a Gamma distribution because the detector works for very few signal samples (such that cannot be assumed to have Gaussian distribution). An analysis of the detector's performance in deriving the test statistic was performed using different values of the power on which the samples are raised as they are summed (a p-norm detector). A generalized ED has been proposed in [45, 46] for different types of fading - mixed distribution of Gamma-distributed variables, Nakagami and Rice distributions. Again the test statistic is compiled from a very small number of samples.

\subsection{Cyclostationary Detectors}

The algorithms of this type are an object of widespread popularity in the context of spectrum sensing in cases where a high detection probability is required at low $\mathrm{SNR}(<0 \mathrm{~dB})$. In addition, the number of samples required to achieve reliability, is much smaller than that of the ED. These advantages are

\footnotetext{
${ }^{2}$ It characterizes the extent to which a CR device contributes to the utilization of the unused frequency resource.
} 


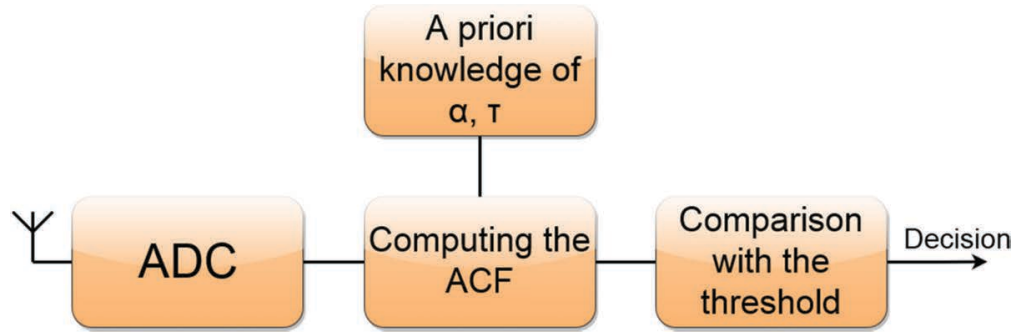

Figure 5 Generalized scheme of the operations in cyclostationary detection-based spectrum sensing.

inherent for this detection technique, which takes advantage of the presence of characteristic cyclostationary features in communication signals. Noise differs from the PU signal in the sense that it does not contain these features due to its non-harmonic structure. Most often, these cyclostationary features are the time delay $\tau$ and the cyclic frequency $\alpha$, which are determined by the structure of the carrier signal, i.e. their values through standards. These features are maintained even if the noise level is much higher than that of the signal. Thus, the decision will be made on the basis of their presence or absence. In general, the test statistic $\Lambda_{\tau}(y)$ of the cyclostationary detector (CD) is defined as the autocorrelation function (ACF) of the received signal samples:

$$
\Lambda_{\tau}(y)=\left|R_{y}(\tau)\right|^{2}=\left|\sum_{i=0}^{N-\tau-1} y[i] y^{*}[i+\tau]\right|^{2}
$$

The structure comprised of the general building blocks of this detector are given in Figure 5.

Theoretically, the ACF of the noise will be 0 , but in real cases and simulation studies, its value is likely to be close to 0 , but still higher. For this reason, as with the other detectors, a decision threshold is introduced, most commonly defined by the CFAR method. Different ACF variants are applied to $\mathrm{CDs}$ to achieve the right trade-off between computational complexity and efficiency. One such test statistic variant is the absolute value of the sum of the covariance matrix of the received signal [49]. The threshold used for the decision on spectrum occupancy is derived through the proposed Covariance Absolute Value model.

The spectral correlation function (SCF) is the most commonly applied ([50-52]) test statistic for one (single cycle, SC) or multiple cyclic frequencies (multi-cycle, MC) of the PU signal. SCF is composed of the signal power for one or more cyclic frequencies $\alpha_{k}$, i.e. the cyclic autocorrelation function 
(CAF) at $\tau=0$, which is a variant of the ACF [53]:

$$
\begin{aligned}
& \Lambda_{S C}(y)=\left|R_{y}^{\alpha}(\tau=0)\right|^{2}=\left|\sum_{i=0}^{N-1} y[i]^{2} e^{-j 2 \pi \alpha n}\right|^{2}, \\
& \Lambda_{M C}(y)=\left|\sum_{k=1}^{N_{\alpha}} R_{y}^{\alpha_{k}}(\tau=0)\right|^{2},
\end{aligned}
$$

Usually, the Gaussian noise assumption for expressing $P_{D}$ and the threshold value, holds. The [50] method offers a cooperative spectrum sensing scenario in which each SU in a CR network calculates the SCF at one of the PU's cyclic signal frequencies, with the decision center combining their results to assess spectrum availability. In this way, the computational complexity of the SCF is reduced in proportion to the number of devices on the network. This study, in addition, considers the effect of log-normal shadowing. As an alternative, to achieve lower computational complexity, instead of SCF, the CAF can be used for a standard signal cyclic frequency and time delay [53]:

$$
\Lambda_{\tau, \alpha}(y)=\left|R_{y}^{\alpha}(\tau)\right|^{2}=\left|\sum_{i=0}^{N-\tau-1} y[i] y^{*}[i+\tau] e^{-j 2 \pi \alpha n}\right|^{2}
$$

Aside from the Gaussian distribution of the noise, [53] considers Rayleigh fading. The study in [54] examines, in addition to the noise, the influence of flat fading and defines test statistics through the coherent spectrum function, which is a normalized form of the SCF. The method proposed in [55] examines the CD influenced by log-normal shadowing. In [56], the signal is pre-processed through compressed sensing in order to detect the broadband signal more effectively. Instead of an explicitly defined threshold, this study utilizes the extraction of CAF at specific cyclic frequencies. If the highest values of the CAF are the same or close, the PU is present.

\subsection{Eigenvalue-based Detectors}

In essence, such approaches are based on various kinds of matrix decomposition of the received samples. Its advantage is that it does not need a priori information on signal and noise distribution, while at the same time the mathematical description of the detector is simpler than that of the $\mathrm{CD}$. The disadvantage of the eigenvalue-based detectors (EBD) is the need for performing matrix operations which have considerable computational complexity. 


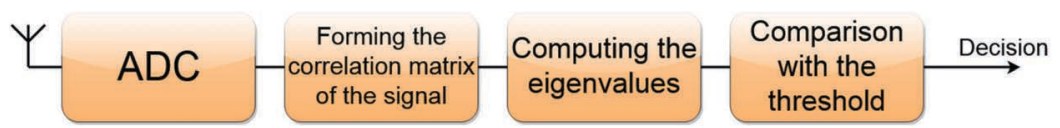

Figure 6 Generalized scheme of the operations in eigenvalue-based spectrum sensing.

The test statistic's derivation for this type of detector is based on the following operations. ${ }^{3}$ The vector of received $\mathrm{N}$ samples is divided into $\mathrm{L}$ equal parts that form the $\mathbf{Y}$ matrix of dimensions $L \times N_{L}$, where $N_{L}=\frac{N}{L}$ [39]:

$$
\mathbf{Y}=\left[\begin{array}{ccccc}
y_{1}[0] & y_{1}[1] & y_{1}[2] & \cdots & y_{1}\left[N_{L}\right] \\
y_{2}[0] & y_{2}[1] & y_{2}[2] & \cdots & y_{2}\left[N_{L}\right] \\
\vdots & \vdots & \vdots & \vdots & \vdots \\
y_{L}[0] & y_{L}[1] & y_{L}[2] & \cdots & y_{L}\left[N_{L}\right]
\end{array}\right]
$$

The covariance matrix of $\mathbf{Y}$ is defined as follows $[39,57]$ :

$$
\mathbf{R}_{r}=\frac{1}{N_{L}} \mathbf{Y Y}^{*}
$$

The test statistic of the EBD is formed by the eigenvalues of $\mathbf{R}_{r}$. Generally, it is defined as the ratio between the largest $\delta_{\max }$ and smallest $\delta_{\min }$ eigenvalues (also known as Maximum to Minimum Eigenvalue method or MME):

$$
\Lambda_{\delta}(y)=\frac{\delta_{\max }}{\delta_{\min }}
$$

If this ratio is equal to 1 , then that is because both eigenvalues contain just the noise variance $\sigma_{n}^{2}$. In the alternative case, it will be higher than 1 and the presence of the PU signal will be detected. The scheme of operation of the EBD is illustrated in Figure 6.

The EBD approach is applied in $[39,58]$ but instead of the standard assumption for the threshold equal to one, it is derived by the CFAR method. In addition, a variant of the test statistics is introduced in which the average signal power is divided by $\delta_{\min }$ (Energy to Minimum Eignevalue, EME). Authors in [58] develop an optimal threshold for guaranteed values of both $P_{D}$ and $P_{F A}$ and specific $N$ (which is a function of the SNR). Gaussian noise is assumed. In [59] a generalized detector model derived from the Neyman-Pearson criterion for unknown values of the noise variance and the

\footnotetext{
${ }^{3}$ It is assumed that a CR device has only one receiving antenna, since the analysis of multiple received signals is beyond the scope of this work.
} 
PU signal's variance is investigated. The test statistic is composed of the ratio between the arithmetic and geometric mean of the eigenvalues. The Gaussian distribution and the presence of noise uncertainty are assumed in [60], where the ratio between $\delta_{\max }$ and $\delta_{\min }$ is used to form $\Lambda_{\delta}(y)$. The study in [61] examines the conditions of a correlated noise channel and a Rayleigh fading using the EME and MME methods. Spectrum sensing is performed by using the SNR adaptive approximation method. An algorithm based on finding the sum of the principal components of $\mathbf{R}_{r}$ of real-time recorded signals, shows better performance than the MME [62].

\subsection{Matched Filter-based Detectors}

This detector achieves optimal signal detection results, but the probability density function (PDF) of the PU signal must be known a priori or the receiver provided with a coherent demodulator. Despite this limiting factor, the sensing process itself is considerably simplified in terms of computational complexity compared to $\mathrm{CD}$ and EBD methods. In general, the a priori information required for detection is the pilot signal $x_{P}$. The Test statistic and decision threshold are defined as follows [27, 63]:

$$
\begin{aligned}
\Lambda_{P}(y) & =\sum_{i=0}^{N-1}\left|y[k] x_{P}^{*}[i]\right|, \\
\lambda & =Q^{-1}\left(\bar{P}_{F A}\right) \sqrt{P \sigma_{n}^{2}},
\end{aligned}
$$

where $P$ is the average power of the PU signal, while $Q^{-1}($.$) is$ the inverse complimentary distribution function defined as $Q^{-1}(x)=$ $\frac{2 \sqrt{2}}{\pi} \int_{2 x}^{\infty} \exp \left(-t^{2}\right) \mathrm{d} t$. The general structure of the MF-based detector is described in Figure 7.

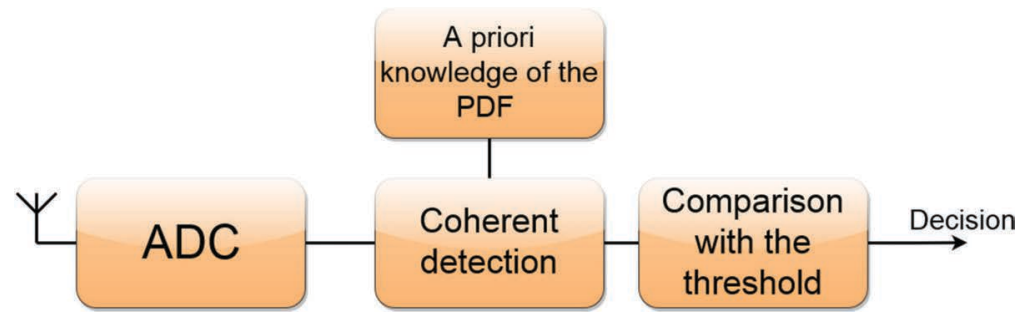

Figure 7 Generalized scheme of the operations in MF-based spectrum sensing. 


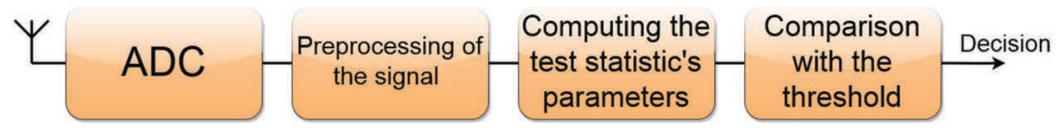

Figure 8 Generalized scheme of the operations in BD-based spectrum sensing.

The effectiveness of MF is shown in the study in [64], where apart from detecting the presence of a signal, a distinction is made between the power levels of the received signals. In addition, the matched filter shows its superiority over ED in processing real recorded signals for a relatively small number of samples in [36]. MF achieves a $90 \%$ detection probability even at very low SNR levels $(<-20 \mathrm{~dB})$ for Gaussian noise assumption.

\subsection{Blind Detectors}

These detectors are not characterized by the need for specific a priori information in order to perform their function. This makes them widely applicable in scenarios where the receiver is not designed to operate in a predetermined frequency range but must dynamically calculate the parameters of signals with unknown distributions. These algorithms have a higher computational complexity than most of the others due to the pre-processing required before the test statistics are generated. The general operation scheme of the blind detector (BD) is shown in Figure 8.

The approach in [65] applies an inverse Fourier transform and obtains the cyclic spectral density of the CAF (which includes another Fourier transform) to estimate the occupancy of the signal spectrum. The authors in [66] propose a BD based on Fast Fourier Transform Accumulation for orthogonal frequency division multiplexed (OFDM) signals. It involves the decimation and separation of the signal into fragments on which a Fourier transform is performed to estimate the SCF so that the CD-based sensing can be applied. High-probability detection at low SNR is achieved in [67], where the signal is pre-processed by the compressed sensing method. The test statistic is formed by the symmetry index, which is defined as the average of all cyclic frequencies. The threshold value is determined empirically. All of these BD-based studies consider Gaussian noise.

\subsection{Others}

This category includes algorithms that apply test statistic determination techniques which differ significantly from the other five types, often based 
on or combining two or more of them. The mathematical derivation of these approaches differs significantly from the rest and reducing the feature extraction tasks they solve to any of the more traditional categories would be difficult. In view of this, their computational complexity also varies greatly.

A substantial portion ([34, 40,52, 68-70]) of the algorithms in this group, introduce a hybrid sequence of different spectrum sensing methods and logical decision making operations. In [40], a hybrid method is proposed that combines ED and $\mathrm{CD}$. If the signal level is below a certain value, which will result in a lower $P_{D}$, then the $\mathrm{CD}$ is enabled to evaluate the spectrum occupancy. Otherwise, the decision is made by the faster ED. Variants of this approach are explored in [52, 70]. Similarly, [34] combines ED and EBDbased methods whose test statistics are composed of the highest eigenvalue, covariance, or MME. In relation to noise uncertainty, [68] proposes combining standard ED with a method which uses the Akaike information criterion based on singular matrix decomposition. It will be utilized if the decision made by the ED does not reach the desired reliability. The method proposed in [69] combines ED, CD, and MF solutions with fuzzy logic (FL) to achieve a higher detection probability. The result is converted to a numerical value to be compared with the decision threshold.

Detection in Cauchy noise is examined in [71]. A constant threshold value is determined by which to compare the differential entropy of the signal samples (method for estimating the PDF). The detector is effective for low number of samples $(<100)$ and low value of the guaranteed $P_{F A}(<10 \%)$. Wideband sensing is achieved for low SNR $(<0 \mathrm{~dB})$ through a multi-stage Wiener filter in [72]. The test statistic is formed by the method of minimizing the root mean square error. An alternative approach for wideband sensing is presented in [73]. Compressed sensing based on wavelet transformation is applied on the broadband signal, after which the compressed signal's power for 10000 samples is compared to the threshold value. [74], a detector is proposed that makes a decision on the basis excess and asymmetry of signal samples (Jarque-Bera test statistic), achieving effective detection for very low SNR levels $(<-15 \mathrm{~dB})$ and the presence of Rayleigh fading.

\section{Summary and Challenges for Future Research}

The hereby presented review outlines several tendencies in recent local spectrum sensing research. They are as follows:

- Prevalent standard assumptions for the channel model - the vast majority of published works on spectrum sensing do not consider complex fading 
channels with non-Gaussian assumption for the signal and noise due to the increased difficulty in the test statistic's derivation. There is some evidence [75-80] that empirically-collected measurements are not always described properly by Nakagami/Rayleigh fading and Gaussian noise. In addition, any of the examined detector types is best suited for a particular application due to complexity in mathematical definition, computational requirements, need of a priori information and implementation considerations. However, most of the studies are based on the ED due to its speed which is essential in CR development.

- Explicit definition of a numerical threshold as a decision-making logicit is the almost universally used metric for spectrum occupancy determination. The main difficulty in practice, is defining its value which is based on the noise power. In addition, depending on the specific use-case, it may be necessary for the threshold to be adaptively changed to accommodate for interfering signals from the PU's point of view.

- The execution speed of the sensing algorithm and the factors that determine it, are very rarely discussed. A typical aspect of every detection approach is the optimization of its two conflicting main parameters $P_{F A}$ and $P_{D}$. This is a central problem in designing the sensing algorithm because its solution is usually dependent on the choice of decision threshold and $t_{S}$ which also need to be optimized for efficiency.

Based on this outline, the following research directions can be defined:

- Inclusion of more general channel models in the development of signal detectors is necessitated by the realistic propagation environment which is characterized by spatial correlation of the surfaces and non-Gaussian interference created by unwanted transmissions of electronic equipment. This is specifically important for ED-based algorithms because they rely on noise estimation to form their test statistic. Additionally, recent findings demonstrate that the wireless channel in UDNs differs much from the traditional cellular one. It is characterized by significant increase in interference power from neighboring access points as their densification increases, and non-linear dependency of the path loss from the distance [81].

- Further study is needed to determine the choice of efficient detector in dense 5G systems such as UDN and D2D. Thus is the analysis expanded from sensing in the time and frequency to include the spatial domain [82]. It plays a role in dense CR networks because identifying spectrum 
holes at a certain location leads to a greater protection of the PU against unwanted interference.

- The recently developed non-orthogonal multiple access (NOMA) has been recognized as a very promising solution for spectrum sensing in future CRs. It allows both overlay and underlay deployments of a CR network due to multiplexing the user traffic in the power domain in addition to time and frequency [83]. The problems related to finding an optimal balance between the conflicting detection parameters $\left(P_{F A}\right.$, $P_{D}$ and $t_{S}$ ) are still relevant for NOMA-based CR systems [84]. This naturally leads to the efficiency-accuracy trade-off problem $[85,86]$ which has been an object of intensive research effort in the recent years. It expands the optimization task to include the transmission period of the SU if the spectrum is unoccupied.

- Identification of the signal's source - it can extend the sensing function to allow for classification of the signal's modulation through machine learning-based algorithms. Thus, the transmitter's type or location can be identified. While reliable detection is required at very low SNR, classification may only be necessary for SNR levels over $0 \mathrm{~dB}$ to detect interferers from the point of view of the PU, or other CR users. This clarification is motivated by the significant decline in accuracy of the available classifiers when the noise levels are close to or higher than that of the signal $[87,88]$.

- The CR system's reliance on spectrum occupancy estimation makes it vulnerable to attacks at the physical level. If an attacker device with cognitive capabilities is not properly identified, it may attempt to eavesdrop the SU's communications or disrupt them by exploiting the spectrum. Physical layer security can be complemented by the signal sensing and classification functionality, or during transmission [89].

- Efficient spectrum regulation through long-term monitoring - in addition to the usual operation of CR devices, spectrum sensing is also important for analysis of large volumes of recorded signal data (Big Data RF analytics) for the purpose of obtaining a better depiction of the spectrum usage in a certain area $[90,91]$. Thus, poor utilization, unwanted transmissions and the reasons for their occurrence can be identified much more accurately. The cognition-enabled equipment with its capability to estimate the location and type (from which other parameters of the signal's source can be inferred because they will likely be standardized) of the transmitter can greatly benefit the long-term spectrum monitoring service. 
This review discusses the potential scenarios in which CR functionalities can be integrated into the future $5 \mathrm{G}$ networks. Through such technological convergence, these systems can be properly deployed alongside the legacy communication standards which occupy the bands under $6 \mathrm{GHz}$. As an enabler for this integration, the major types of spectrum sensing methods as well as the characteristics of their system models are also specified. As a consequence of the observed trends, the areas for future advancement of local spectrum sensing are outlined.

\section{Acknowledgments}

This work was supported by research project KP-06-N27/3/08.12.2018 "Resource self-configuration and management in ultra-dense networks with user centric wireless access" of the Bulgarian Research Fund of the Ministry of Education and Science.

\section{References}

[1] S. Orts-Escolano, C. Rhemann, S. Fanello, W. Chang, A. Kowdle, Y. Degtyarev, D. Kim, P. L. Davidson, S. Khamis, M. Dou et al., "Holoportation: Virtual 3D teleportation in real-time," in Proceedings of the 29th Annual Symposium on User Interface Software and Technology. ACM, 2016, pp. 741-754.

[2] A. Osseiran, F. Boccardi, V. Braun, K. Kusume, P. Marsch, M. Maternia, O. Queseth, M. Schellmann, H. Schotten, H. Taoka et al., "Scenarios for $5 \mathrm{G}$ mobile and wireless communications: the vision of the metis project," IEEE Communications Magazine, vol. 52, no. 5, pp. 26-35, 2014.

[3] M. Agiwal, A. Roy, and N. Saxena, "Next generation 5G wireless networks: A comprehensive survey," IEEE Communications Surveys \& Tutorials, vol. 18, no. 3, pp. 1617-1655, 2016.

[4] T. Reuters, "The world in 2025: 10 predictions of innovation," 2014.

[5] C. V. N. Index, "Global mobile data traffic forecast update, 2017-2022 white paper," link: http://goo.gl/ylTuVx, 2019.

[6] M. R. Dzulkifli, M. R. Kamarudin, and T. A. Rahman, "Spectrum occupancy at UHF TV band for cognitive radio applications," in 2011 IEEE International RF \& Microwave Conference. IEEE, 2011, pp. 111-114. 
[7] M. H. Islam, C. L. Koh, S. W. Oh, X. Qing, Y. Y. Lai, C. Wang, Y.-C. Liang, B. E. Toh, F. Chin, G. L. Tan et al., "Spectrum survey in Singapore: Occupancy measurements and analyses," in 2008 3rd International Conference on Cognitive Radio Oriented Wireless Networks and Communications (CrownCom 2008). IEEE, 2008, pp. 1-7.

[8] K. Patil, R. Prasad, and K. Skouby, "A survey of worldwide spectrum occupancy measurement campaigns for cognitive radio," in 2011 International Conference on Devices and Communications (ICDeCom). IEEE, 2011, pp. 1-5.

[9] S. Haykin, "Cognitive radio: brain-empowered wireless communications," IEEE journal on selected areas in communications, vol. 23, no. 2, pp. 201-220, 2005.

[10] A. Ivanov, K. Tonchev, V. Poulkov, and A. Manolova, "Framework for implementation of cognitive radio based ultra-dense networks," in 2019 42nd International Conference on Telecommunications and Signal Processing (TSP). IEEE, 2019, pp. 481-486. DOI: 10.1109/TSP.2019.8 769067

[11] F. Tseng, L. Chou, H. Chao, and J. Wang, "Ultra-dense small cell planning using cognitive radio network toward 5G," IEEE Wireless Communications, vol. 22, no. 6, pp. 76-83, December 2015.

[12] S. Chen, F. Qin, B. Hu, X. Li, and Z. Chen, "User-centric ultra-dense networks for 5G: challenges, methodologies, and directions," IEEE Wireless Communications, vol. 23, no. 2, pp. 78-85, April 2016.

[13] Z. Zhang, W. Zhang, S. Zeadally, Y. Wang, and Y. Liu, "Cognitive radio spectrum sensing framework based on multi-agent arc hitecture for $5 \mathrm{G}$ networks," IEEE Wireless Communications, vol. 22, no. 6, pp. 34-39, December 2015.

[14] A. Al-Dulaimi, S. Al-Rubaye, J. Cosmas, and A. Anpalagan, "Planning of ultra-dense wireless networks," IEEE Network, vol. 31, no. 2, pp. 90-96, March 2017.

[15] S. A. R. Zaidi, D. C. McLernon, M. Ghogho, and M. A. Imran, "Cloud empowered cognitive inter-cell interference coordination for small cellular networks," in 2015 IEEE International Conference on Communication Workshop (ICCW). IEEE, 2015, pp. 2218-2224.

[16] R. Atat, L. Liu, H. Chen, J. Wu, H. Li, and Y. Yi, "Enabling cyberphysical communication in 5G cellular networks: challenges, spatial spectrum sensing, and cyber-security," IET Cyber-Physical Systems: Theory \& Applications, vol. 2, no. 1, pp. 49-54, 2017. 
[17] H. Chen and L. Liu, "Resource allocation for sensing-based device-todevice (D2D) networks," in 2015 49th Asilomar Conference on Signals, Systems and Computers. IEEE, 2015, pp. 1058-1062.

[18] S.-C. Hung, S.-Y. Lien, and K.-C. Chen, "Stochastic topology cognition in heterogeneous networks," in 2013 IEEE 24th International Symposium on Personal, Indoor and Mobile Radio Communications (PIMRC Workshops). IEEE, 2013, pp. 194-199.

[19] A. Al-Dulaimi, S. Al-Rubaye, J. Cosmas, and A. Anpalagan, "Planning of ultra-dense wireless networks," IEEE Network, vol. 31, no. 2, pp. 90-96, 2017.

[20] H. Zhang, L. Song, Y. Li, and G. Y. Li, "Hypergraph theory: Applications in 5G heterogeneous ultra-dense networks," IEEE Communications Magazine, vol. 55, no. 12, pp. 70-76, 2017.

[21] W. Feng, Y. Wang, D. Lin, N. Ge, J. Lu, and S. Li, "When MMWave communications meet network densification: A scalable interference coordination perspective," IEEE Journal on Selected Areas in Communications, vol. 35, no. 7, pp. 1459-1471, 2017.

[22] T. Maksymyuk, M. Brych, Y. Klymash, M. Kyryk, and M. Klymash, "Game theoretical framework for multi-operator spectrum sharing in 5G heterogeneous networks," in 2017 4th International Scientific-Practical Conference Problems of Infocommunications. Science and Technology (PIC S\&T). IEEE, 2017, pp. 515-518.

[23] Y. Wei and S.-H. Hwang, "Optimization of cell size in ultra-dense networks with multiattribute user types and different frequency bands," Wireless Communications and Mobile Computing, vol. 2018, 2018.

[24] C. R. Stevenson, G. Chouinard, Z. Lei, W. Hu, S. J. Shellhammer, and W. Caldwell, "IEEE 802.22: The first cognitive radio wireless regional area network standard," IEEE communications magazine, vol. 47, no. 1, pp. 130-138, 2009.

[25] K.-L. A. Yau, J. Qadir, C. Wu, M. A. Imran, and M. H. Ling, "Cognitioninspired 5G cellular networks: a review and the road ahead," IEEE Access, vol. 6, pp. 35 072-35 090, 2018.

[26] M. Amjad, F. Akhtar, M. H. Rehmani, M. Reisslein, and T. Umer, "Fullduplex communication in cognitive radio networks: A survey," IEEE Communications Surveys \& Tutorials, vol. 19, no. 4, pp. 2158-2191, 2017.

[27] S. M. Kay, "Fundamentals of statistical signal processing - vol. 2: Detection theory," 1998. 
[28] Y.-C. Liang, Y. Zeng, E. Peh, and A. T. Hoang, "Sensing-throughput tradeoff for cognitive radio networks," in 2007 IEEE International Conference on Communications. IEEE, 2007, pp. 5330-5335.

[29] R. K. Dubey and G. Verma, "Improved spectrum sensing for cognitive radio based on adaptive threshold," in 2015 Second International Conference on Advances in Computing and Communication Engineering. IEEE, 2015, pp. 253-256.

[30] A. Bagwari and G. S. Tomar, "Two-stage detectors with multiple energy detectors and adaptive double threshold in cognitive radio networks," International Journal of Distributed Sensor Networks, vol. 9, no. 8, p. $656495,2013$.

[31] S. Shrivastava, R. Tiwari, and S. Das, "Comparative performance evaluation of a new dynamic-double-threshold energy detection scheme with basic spectrum sensing techniques," in 2014 International Conference on Green Computing Communication and Electrical Engineering (ICGCCEE). IEEE, 2014, pp. 1-6.

[32] R. Tandra and A. Sahai, "SNR walls for signal detection," IEEE Journal of selected topics in Signal Processing, vol. 2, no. 1, pp. 4-17, 2008.

[33] Y. H. Chye, E. Dutkiewicz, R. Vesilo, and R. P. Liu, "Adaptive spectrum sensing for cognitive radio systems in a fading environment," in 2014 International Symposium on Wireless Personal Multimedia Communications (WPMC). IEEE, 2014, pp. 451-456.

[34] K. Srisomboon, A. Prayote, and W. Lee, "Two-stage spectrum sensing for cognitive radio under noise uncertainty," in 2015 Eighth International Conference on Mobile Computing and Ubiquitous Networking (ICMU). IEEE, 2015, pp. 19-24.

[35] V. Rakovic, D. Denkovski, V. Atanasovski, P. Mähönen, and L. Gavrilovska, "Capacity-aware cooperative spectrum sensing based on noise power estimation," IEEE Transactions on Communications, vol. 63, no. 7, pp. 2428-2441, 2015.

[36] A. F. Eduardo and R. G. G. Caballero, "Experimental evaluation of performance for spectrum sensing: Matched filter vs energy detector," in IEEE Colombian Conference on Communication and Computing (IEEE COLCOM 2015). IEEE, 2015, pp. 1-6.

[37] L. Hang and F. Takeo, "Single-channel blind identification based advanced energy detection for cognitive radio," in 2016 Eighth International Conference on Ubiquitous and Future Networks (ICUFN). IEEE, 2016, pp. 532-536. 
[38] A. Kaushik, S. K. Sharma, S. Chatzinotas, B. Ottersten, and F. K. Jondral, "Sensing-throughput tradeoff for interweave cognitive radio system: A deployment-centric viewpoint," IEEE Transactions on Wireless Communications, vol. 15, no. 5, pp. 3690-3702, 2016.

[39] A. Nafkha, B. Aziz, M. Naoues, and A. Kliks, "Cyclostationarity-based versus eigenvalues-based algorithms for spectrum sensing in cognitive radio systems: Experimental evaluation using GNU Radio and USRP,' in 2015 IEEE 11th International Conference on Wireless and Mobile Computing, Networking and Communications (WiMob). IEEE, 2015, pp. 310-315.

[40] A. Nafkha, M. Naoues, K. Cichony, A. Kliks, and B. Aziz, "Hybrid spectrum sensing experimental analysis using GNU Radio and USRP for cognitive radio," in 2015 International Symposium on Wireless Communication Systems (ISWCS). IEEE, 2015, pp. 506-510.

[41] S. Atapattu, C. Tellambura, H. Jiang, and N. Rajatheva, "Unified analysis of low-snr energy detection and threshold selection," IEEE Transactions on vehicular technology, vol. 64, no. 11, pp. 5006-5019, 2014.

[42] S. P. Herath, N. Rajatheva, and C. Tellambura, "Energy detection of unknown signals in fading and diversity reception," IEEE Transactions on communications, vol. 59, no. 9, pp. 2443-2453, 2011.

[43] S. Shobitha and S. Gurugopinath, "Energy-based bayesian spectrum sensing over $\alpha-\kappa-\mu$ fading channels," in 2016 IEEE Annual India Conference (INDICON). IEEE, 2016, pp. 1-6.

[44] V. R. S. Banjade, C. Tellambura, and H. Jiang, "Spectrum sensing performance of p-norm detector in random network interference," in 2015 IEEE International Conference on Communications (ICC). IEEE, 2015, pp. 7474-7479.

[45] H. Huang, "Performance evaluation of energy detector over generalized non-linear and shadowed composite fading channels using a mixture gamma distribution," arXiv preprint arXiv:1707.07849, 2017.

[46] S. P. Herath, N. Rajatheva, T. Le-Ngoc, and C. Tellambura, "Energy detection with diversity reception," Journal of Science and Technology: Issue on Information and Communications Technology, vol. 3, no. 1, pp. 20-28, 2017.

[47] S. Gurugopinath, "Energy-based bayesian spectrum sensing over $\alpha-\mu /$ stacy/generalized gamma fading channels," in Communication Systems and Networks (COMSNETS), 2016 8th International Conference on. IEEE, 2016, pp. 1-6. 
[48] P. K. Varshney, Distributed detection and data fusion. Springer Science \& Business Media, 2012.

[49] Y. Zeng and Y.-C. Liang, "Spectrum-sensing algorithms for cognitive radio based on statistical covariances," IEEE transactions on Vehicular Technology, vol. 58, no. 4, pp. 1804-1815, 2008.

[50] M. Derakhshani, T. Le-Ngoc, and M. Nasiri-Kenari, "Efficient cooperative cyclostationary spectrum sensing in cognitive radios at low SNR regimes," IEEE Transactions on wireless communications, vol. 10, no. 11, pp. 3754-3764, 2011.

[51] A. Bagwari and B. Singh, "Comparative performance evaluation of spectrum sensing techniques for cognitive radio networks," in 2012 Fourth International Conference on Computational Intelligence and Communication Networks. IEEE, 2012, pp. 98-105.

[52] D. Han and H. Liu, "An energy detection based on cyclostationary," in 2011 7th International Conference on Wireless Communications, Networking and Mobile Computing. IEEE, 2011, pp. 1-4.

[53] A. Tani, R. Fantacci, and D. Marabissi, "A low-complexity cyclostationary spectrum sensing for interference avoidance in femtocell LTE-A-based networks," IEEE Transactions on Vehicular Technology, vol. 65, no. 4, pp. 2747-2753, 2015.

[54] D. Li, L. Zhang, Z. Liu, Z. Wu, and Z. Zhang, "Mixed signal detection and carrier frequency estimation based on spectral coherent features," in 2016 International Conference on Computing, Networking and Communications (ICNC). IEEE, 2016, pp. 1-5.

[55] M. Derakhshani, M. Nasiri-Kenari, and T. Le-Ngoc, "Cooperative cyclostationary spectrum sensing in cognitive radios at low SNR regimes," in 2010 IEEE International Conference on Communications, May 2010, pp. 1-5.

[56] V. Ramachandran and A. Cheeran, "Improvement of energy efficiency of spectrum sensing algorithms for cognitive radio networks using compressive sensing technique," in 2014 International Conference on Computer Communication and Informatics. IEEE, 2014, pp. 1-6.

[57] Y. Zeng and Y.-C. Liang, "Maximum-minimum eigenvalue detection for cognitive radio," in 2007 IEEE 18th International Symposium on Personal, Indoor and Mobile Radio Communications. IEEE, 2007, pp. 1-5.

[58] C. Charan and R. Pandey, "Eigenvalue-based reliable spectrum sensing scheme for cognitive radio networks," in 2017 International Conference on Nascent Technologies in Engineering (ICNTE). IEEE, 2017, pp. 1-5. 
[59] T. J. Lim, R. Zhang, Y. C. Liang, and Y. Zeng, "GLRT-based spectrum sensing for cognitive radio," in IEEE GLOBECOM 2008 - 2008 IEEE Global Telecommunications Conference, Nov. 2008, pp. 1-5.

[60] C. Jiang, Y. Li, W. Bai, Y. Yang, and J. Hu, "Statistical matched filter based robust spectrum sensing in noise uncertainty environment," in 2012 IEEE 14th International Conference on Communication Technology. IEEE, 2012, pp. 1209-1213.

[61] S. K. Sharma, S. Chatzinotas, and B. Ottersten, "SNR estimation for multi-dimensional cognitive receiver under correlated channel/noise," IEEE Transactions on Wireless Communications, vol. 12, no. 12, pp. 6392-6405, 2013.

[62] Z. Idrees, F. A. Bhatti, and A. Rashdi, "Spectrum sensing using lowcomplexity principal components for cognitive radios," EURASIP Journal on Wireless Communications and Networking, vol. 2015, no. 1, p. 184, 2015.

[63] F. Salahdine, H. El Ghazi, N. Kaabouch, and W. F. Fihri, "Matched filter detection with dynamic threshold for cognitive radio networks," in 2015 International Conference on Wireless Networks and Mobile Communications (WINCOM). IEEE, 2015, pp. 1-6.

[64] X. Zhang, R. Chai, and F. Gao, "Matched filter based spectrum sensing and power level detection for cognitive radio network," in 2014 IEEE global conference on signal and information processing (GlobalSIP). IEEE, 2014, pp. 1267-1270.

[65] W. M. Jang, "Blind cyclostationary spectrum sensing in cognitive radios," IEEE Communications Letters, vol. 18, no. 3, pp. 393-396, 2014.

[66] G. Prema and P. Gayatri, "Blind spectrum sensing method for OFDM signal detection in cognitive radio communications," in 2014 International Conference on Communication and Network Technologies. IEEE, 2014, pp. 42-47.

[67] L. Safatly, B. Aziz, A. Nafkha, Y. Louet, Y. Nasser, A. El-Hajj, and K. Y. Kabalan, "Blind spectrum sensing using symmetry property of cyclic autocorrelation function: from theory to practice," EURASIP Journal on Wireless Communications and Networking, vol. 2014, no. 1, p. 26, 2014.

[68] P. P. Anaand and C. Charan, "Two stage spectrum sensing for cognitive radio networks using ED and AIC under noise uncertainty," in 2016 International Conference on Recent Trends in Information Technology (ICRTIT). IEEE, 2016, pp. 1-6. 
[69] W. Ejaz, N. ul Hasan, S. Aslam, and H. S. Kim, "Fuzzy logic based spectrum sensing for cognitive radio networks," in 2011 Fifth International Conference on Next Generation Mobile Applications, Services and Technologies. IEEE, 2011, pp. 185-189.

[70] Z. Khalaf, A. Nafkha, and J. Palicot, "Enhanced hybrid spectrum sensing architecture for cognitive radio equipment," in 2011 XXXth URSI General Assembly and Scientific Symposium. IEEE, 2011, pp. 1-4.

[71] S. Gurugopinath, R. Muralishankar, and H. Shankar, "Spectrum sensing in the presence of cauchy noise through differential entropy," in 2016 IEEE Distributed Computing, VLSI, Electrical Circuits and Robotics (DISCOVER). IEEE, 2016, pp. 201-204.

[72] A. Badarudeen and K. Gopakumar, "Wideband spectrum sensing using multi stage weiner filter," in 2016 IEEE 7th Annual Ubiquitous Computing, Electronics \& Mobile Communication Conference (UEMCON). IEEE, 2016, pp. 1-5.

[73] N. Kumar and N. Sood, "Fast and efficient compressive sensing for wideband cognitive radio systems," in 2015 IEEE 2nd International Conference on Recent Trends in Information Systems (ReTIS). IEEE, 2015, pp. 87-91.

[74] A. Subekti, A. B. Suksmono et al., "A Jarque-Bera test based spectrum sensing for cognitive radio," in 2014 8th International Conference on Telecommunication Systems Services and Applications (TSSA). IEEE, 2014, pp. 1-4.

[75] G. Fraidenraich and M. D. Yacoub, "The $\alpha-\eta-\mu$ and $\alpha-\kappa-\mu$ fading distributions," in 2006 IEEE Ninth International Symposium on Spread Spectrum Techniques and Applications. IEEE, 2006, pp. 16-20.

[76] S. A. Bhatti, Q. Shan, I. A. Glover, R. Atkinson, I. E. Portugues, P. J. Moore, and R. Rutherford, "Impulsive noise modelling and prediction of its impact on the performance of WLAN receiver," in Signal Processing Conference, 2009 17th European. IEEE, 2009, pp. 1680-1684.

[77] P. G. Georgiou, P. Tsakalides, and C. Kyriakakis, "Alpha-stable modeling of noise and robust time-delay estimation in the presence of impulsive noise," IEEE transactions on multimedia, vol. 1, no. 3, pp. 291-301, 1999.

[78] D. Middleton, "Non-Gaussian noise models in signal processing for telecommunications: new methods an results for class a and class $b$ noise models," IEEE Transactions on Information Theory, vol. 45, no. 4, pp. 1129-1149, 1999. 
[79] A. Spaulding and D. Middleton, "Optimum reception in an impulsive interference environment - part I: Coherent detection," IEEE Transactions on Communications, vol. 25, no. 9, pp. 910-923, September 1977.

[80] P. Tsakalides and C. L. Nikias, "Maximum likelihood localization of sources in noise modeled as a stable process," IEEE Transactions on Signal Processing, vol. 43, no. 11, pp. 2700-2713, 1995.

[81] J. Liu, M. Sheng, L. Liu, and J. Li, "Network densification in 5G: From the short-range communications perspective," IEEE Communications Magazine, vol. 55, no. 12, pp. 96-102, 2017.

[82] H. Chen, L. Liu, T. Novlan, J. D. Matyjas, B. L. Ng, and J. Zhang, "Spatial spectrum sensing-based device-to-device cellular networks," IEEE Transactions on Wireless Communications, vol. 15, no. 11, pp. 7299-7313, 2016.

[83] L. Lv, J. Chen, Q. Ni, Z. Ding, and H. Jiang, "Cognitive non-orthogonal multiple access with cooperative relaying: A new wireless frontier for 5G spectrum sharing," IEEE Communications Magazine, vol. 56, no. 4, pp. 188-195, 2018.

[84] X. Liu, Y. Wang, S. Liu, and J. Meng, "Spectrum resource optimization for noma-based cognitive radio in $5 \mathrm{G}$ communications," IEEE Access, vol. 6, pp. 24 904-24 911, 2018.

[85] A. Ivanov, A. Mihovska, K. Tonchev, and V. Poulkov, "Real-time adaptive spectrum sensing for cyclostationary and energy detectors," IEEE Aerospace and Electronic Systems Magazine, vol. 33, no. 5-6, pp. 20-33, 2018. DOI: 10.1109/MAES.2018.170098

[86] W.-Y. Lee and I. F. Akyildiz, "Optimal spectrum sensing framework for cognitive radio networks," IEEE Transactions on wireless communications, vol. 7, no. 10, pp. 3845-3857, 2008.

[87] T. J. O'Shea, T. Roy, and T. C. Clancy, "Over-the-air deep learning based radio signal classification," IEEE Journal of Selected Topics in Signal Processing, vol. 12, no. 1, pp. 168-179, 2018.

[88] A. Ivanov, K. Tonchev, V. Poulkov, H. Al-Shatri, and A. Klein, "Hybrid noise-resilient deep learning architecture for modulation classification in cognitive radio networks," in International Conference on Future Access Enablers of Ubiquitous and Intelligent Infrastructures. Springer, 2019, pp. 214-227. DOI: https://doi.org/10.1007/978-3-030-23976-3_20

[89] P. Singh, P. Pawar, and A. Trivedi, "Physical layer security approaches in 5G wireless communication networks," in 2018 First 
International Conference on Secure Cyber Computing and Communication (ICSCCC). IEEE, 2018, pp. 477-482.

[90] T. Cooklev, V. Poulkov, D. Bennett, and K. Tonchev, "Enabling RF data analytics services and applications via cloudification," IEEE Aerospace and Electronic Systems Magazine, vol. 33, no. 5-6, pp. 44-55, 2018. DOI: 10.1109/MAES.2018.170108

[91] P. Baltiiski, I. Iliev, B. Kehaiov, V. Poulkov, and T. Cooklev, "Long-term spectrum monitoring with big data analysis and machine learning for cloud-based radio access networks," Wireless Personal Communications, vol. 87, no. 3, pp. 815-835, 2016. DOI: https://doi.org/10.1007/s11277-015-2631-8

\section{Biography}

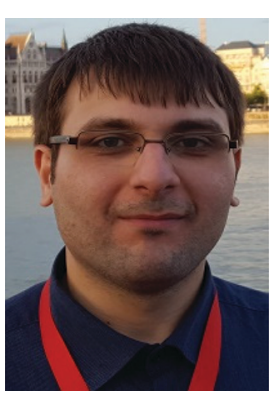

Antoni Ivanov obtained his Master in Innovative Communication Technologies and Entrepreneurship from Technical University of Sofia, Bulgaria and Aalborg University, Denmark in 2016. He is currently working toward his $\mathrm{PhD}$ at the Department of Communication Networks, Faculty of Telecommunications of the Technical University of Sofia. His research interests include cognitive ultra-dense networks, adaptive algorithms for dynamic spectrum access, deep learning-based solutions for cognitive radio applications, and spectral efficiency in the spatial and volume domains for $5 \mathrm{G}$ and beyond. 
